\title{
Ovarian Carcinosarcoma
}

Fouzia El Hilali ${ }^{1 *}$, Ezza Lemrabott ${ }^{1}$, Sanaa Errarhay ${ }^{1}$, Nissrine Mamouni ${ }^{1}$, Chahrazed Bouchikhi ${ }^{1}$, Abdelaziz Banani ${ }^{1}$

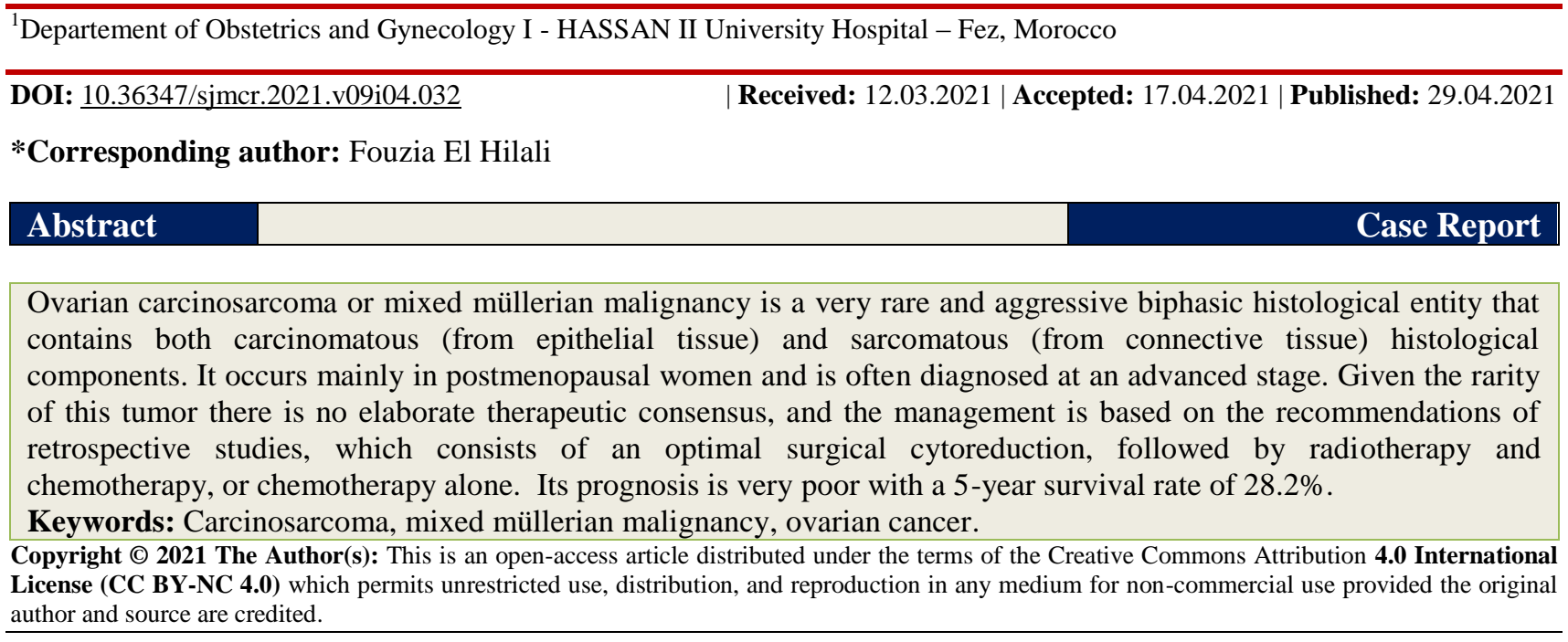

\section{INTRODUCTION}

Ovarian carcinosarcoma (OCS) also known as mixed müllerian malignancy is a very rare and extremely aggressive biphasic histological entity that contains both carcinomatous tissue (from epithelial tissue) and sarcomatous tissue (mesenchymal, from connective tissue) [1]. It represents less than $1 \%$ of all ovarian malignancies [2]. Its prognosis is poor with a disease-specific survival rate of $28.2 \%$ at five years [3].

\section{ObServation}

Female patient, 75 years old, Gravida 2 Para 1, menopausal, with an increase in abdominal volume and abdominopelvic pain that has been evolving for two years. Clinical examination found an abdomino-pelvic mass reaching the umbilicus, with a macroscopically normal cervix without metrorrhagia. On pelvic ultrasound: latero-uterine echogenic image containing multilocular areas in some parts, this mass measures $15 \times 10 \mathrm{~cm}$, and is prolonged below by another cystic mass of $8 \mathrm{~cm}$ in diameter.
Abdomino Pelvic Computed Tomography (AP CT) showed an abdominopelvic mass with a mixed tissue and cystic component measuring $17 \times 12 \mathrm{~cm}$ (Figure-1). Pelvic Magnetic Resonance Imaging (MRI) revealed a large multilocular cystic mass measuring 16 $\mathrm{cm}$ in diameter with solid components and vegetations, evocative of a tumor mass with an ovarian origin (Figure-2).

The patient underwent a median laparotomy with a voluminous mass in the right ovary measuring 30 $\mathrm{x} 10 \mathrm{~cm}$ with a double component: tissue and cystic. A hysterectomy and bilateral adnexectomy were performed, with infracolic omentectomy and multiple peritoneal biopsies. The histological examination concluded to a mixed malignant mullerian tumor of the right ovary with for the epithelial component: high grade serous adenocarcinoma, and for the mesenchymal component: heterologous chondrosarcomatous differentiation. The patient received adjuvant chemotherapy.

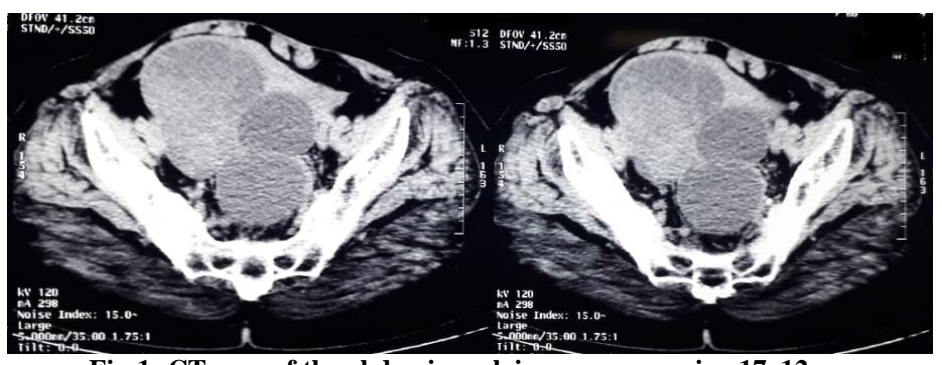

Fig-1: CT scan of the abdominopelvic mass measuring $17 \times 12 \mathrm{~cm}$ 


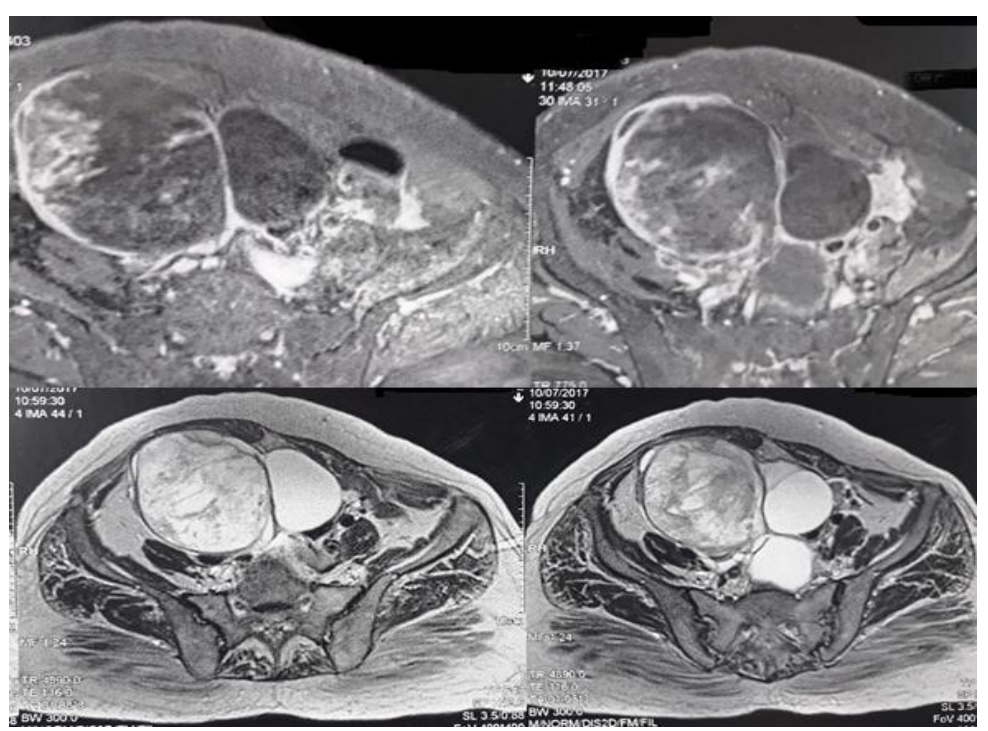

Fig-2: MRI appearance of ovarian mass of multilocular cystic nature measuring $16 \mathrm{~cm}$ in large diameter with solid components and vegetations

\section{DiscuSSION}

OCS occurs in postmenopausal women with an average age of 65 years, diagnosed in $90 \%$ of cases at an advanced stage [4]. They are clinically manifested by abdominopelvic pain, abdominal distension and are sometimes associated with digestive disorders, with an abdominopelvic mass on clinical examination.

These tumors are usually very large in diameter, ranging from 10 to $20 \mathrm{~cm}$, solid-cystic, and most often extend beyond the ovary and into the peritoneum. They contain two components, one carcinomatous (originating from the epithelial tissue) and the other sarcomatous (mesenchymal, originating from the connective tissue). These two components of the OCS are usually mixed randomly [5].

The most frequently observed epithelial
components are serous, endometrioid, or
undifferentiated adenocarcinomas. OCSs are classified
according to homologous or heterologous derivation of
mesenchymal tissue into their stromal component.
Homologous carcinosarcomas contain sarcomatous
elements that differentiate from physiologically native
ovarian tissue and include fibrosarcoma and
leiomyosarcoma. Heterologous components usually
contain malignant osteoid, chondroid or rhabdomyoid
cells that are physiologically foreign to the primary site
[6]. In our case it is a heterologous carcinosarcoma.

There is no international consensus for the management of OCS due to its rarity, and generally the therapeutic approach follows the recommendations of retrospective studies, and consists of an optimal surgical cytoreduction including hysterectomy, bilateral adnexectomy, omentectomy, and appendectomy, with lumbo-aortic and pelvic lymphadenectomy, and multiple peritoneal biopsies [5].
The surgery is followed by both radiotherapy and chemotherapy or chemotherapy alone. Chemotherapy may be based on platinum salts alone, or a combination of carboplatin-paclitaxel or platinumifosfamide [1]. In some cases, three or six cycles of chemotherapy may be given preoperatively to achieve an optimal surgical outcome [5].

The prognosis of OCS is unfavorable compared to epithelial carcinoma of the ovary with a median overall survival ranging from 7 to 27 months [7], according to the review of the literature by Boussios et al, several prognostic factors have been described, including age less than 65 years, disease stage, a preponderant sarcomatoid component of more than $25 \%$, vascular endothelial growth factor (VEGF) expression, p53 mutation, Ki-67 overexpression, tumor grade and residual disease after surgery [5].

\section{CONCLUSION}

Ovarian carcinosarcoma or malignant mixed Mullerian tumor is a very rare histological entity with a very poor prognosis, whose therapeutic management is based on the recommendations of retrospective studies. More appropriate studies are needed for a better evaluation and the development of a therapeutic consensus.

\section{REFERENCES}

1. Shylasree TS, Bryant A, Athavale R. Chemotherapy and/or radiotherapy in combination with surgery for ovarian carcinosarcoma. Cochrane Database Syst Rev. 2013;2:CD006246.

2. Russell_PB, Solomon_HJ. Malignant mullerian and miscellaneous mesenchymal tumors of ovary ('ovarian sarcomas'). In: Coppleson $\mathrm{M}$ editor(s). Gynecologic Oncology. 2nd Edition. Churchill Livingstone, 1992:971-86. 
3. Rauh-Hain JA, Diver EJ, Clemmer JT, Bradford LS, Clark RM, Growdon WB, Goodman AK, Boruta II DM, Schorge JO, Del Carmen MG. Carcinosarcoma of the ovary compared to papillary serous ovarian carcinoma: a SEER analysis. Gynecologic oncology. 2013 Oct 1;131(1):46-51.

4. Berton-Rigaud, D., Devouassoux-Shisheboran, M., Ledermann, J. A., Leitao, M. M., Powell, M. A., Poveda, A., ... \& Ray-Coquard, I. (2014). Gynecologic Cancer InterGroup (GCIG) consensus review for uterine and ovarian carcinosarcoma. International Journal of Gynecologic Cancer, 24(Supp 3): S55-S60.
5. Boussios, S, Karathanasi A, ZakynthinakisKyriakou N, Tsiouris AK, Chatziantoniou AA., Kanellos FS, Tatsi K. Ovarian carcinosarcoma: Current developments and future perspectives. Critical Reviews in Oncology/Hematology, 2019;134, 46-55.

6. The effect of epithelial and stromal tumor components on FIGO stages III and IV ovarian carcinosarcomas treated with primary surgery and chemotherapy; 1025-1030.

7. Duska LR, Garrett A, Eltabbakh GH, Oliva E, Penson R, Fuller AF. Paclitaxel and platinum chemotherapy for malignant mixed mullerian tumors of the ovary. Gynecologic oncology. 2002 Jun 1;85(3):459-63. 